\title{
INVASIVE FUNGAL INFECTION IN A LEVEL III NICU. (20 YEARS STUDY)
}

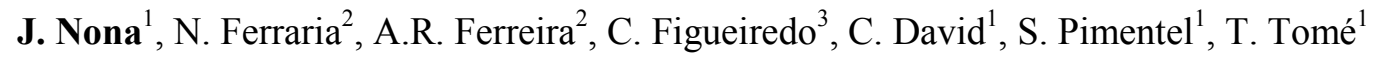 \\ ${ }^{1}$ Departamento de Pediatria - UCIN, Maternidade Alfredo da Costa, Lisboa, ${ }^{2}$ Centro Hospitalar Barreiro \\ Montijo EPE, Barreiro, ${ }^{3}$ HPP-Hospital de Cascais, Cascais, Portugal
}

Background: Invasive fungal infection (IFI) is an important cause of morbidity and mortality in neonatal intensive care units (NICU). New generation lipid formulated amphotericin B has emerged as an efficient and less toxic alternative to treat invasive fungal disease.

Aim: Evaluate epidemiology and risk factors of IFI in very low birth weight neonates (VLBW) in Maternity Alfredo da Costa, Lisbon, Portugal. It's also important to compare the efficacy and adverse effects of treatment with liposomal amphotericin B (L-AmB) and with other forms of amphotericin B (AmP).

Methods: Retrospective study of VLBW patients admitted to a level III NICU between 1990 and 2010 (20 years), with a diagnosis of IFI.

Results: IFI occurred in 23 of estimated 3000 VLBW neonates $(0,8 \%)$; median gestational age was 26 weeks (24-32) and median birth weight was $840 \mathrm{~g}(579-1450)$. The diagnosis was documented by positive blood culture in 20 cases; positive urine culture (bladder catheterization) in 8 cases and autopsy in 4 cases.

Median age at diagnosis was 23 days (10-80). Candida albicans was isolated in 22 patients.

9 patients were treated with L-AmB and 13 patients with other forms of AmP for a median duration of 23 days (2-60). The efficacy rate was $88,9 \%$ for $\mathrm{L}-\mathrm{AmB}$ and $83,3 \%$ for the other forms of AmP.

Conclusions: The incidence of invasive fungal infection in VLBW in our Maternity is similar to other studies. L-AmB had an excellent efficacy rate. This study is a first step to a large prospective multicenter trial. 